\title{
Atomic Non-Locality Dynamics of Two Moving Atoms In A Hybrid Nonlinear System: Concurrence, Uncertainty-Induced Non-Locality And Bell Inequality
}

\author{
A.-B. A. Mohamed \\ Prince Sattam bin Abdulaziz University \\ E.M. Khalil ( $\nabla$ eiedkhalil@yahoo.com ) \\ Taif University College of Science https://orcid.org/0000-0003-0189-845X
}

\section{Research Article}

Keywords: Bell function, skew information quantity, Stark shift, two atoms

Posted Date: June 17th, 2021

DOl: https://doi.org/10.21203/rs.3.rs-581570/v1

License: (c) (1) This work is licensed under a Creative Commons Attribution 4.0 International License.

Read Full License 


\title{
Atomic non-locality dynamics of two moving atoms in a hybrid nonlinear system: Concurrence, uncertainty-induced non-locality and Bell inequality
}

\author{
A.-B. A. Mohamed ${ }^{1,2, *}$ and E.M. Khalil ${ }^{3}$ \\ ${ }^{1}$ Department of Mathematics, College of Science and Humanities in Al-Aflaj, \\ Prince Sattam bin Abdulaziz University, Saudi Arabia \\ ${ }^{2}$ Faculty of Science, Assiut University, Assiut, Egypt \\ ${ }^{3}$ Department of Mathematics, College of Science, \\ P.O. Box 11099, Taif University, Taif 21944, Saudi Arabia.
}

\begin{abstract}
The paper explores the non-locality dynamics of two moving atoms in a hybrid nonlinear system, which describes a nondegenerate parametric amplifier cavity containing two moving atoms with Stark shift interactions. Based on the Bell function, uncertainty-induced non-locality and concurrence, the two moving atomic non-locality is calculated numerically, when the cavity is filled initially by pair coherent fields. The results showed that the hybrid nonlinear qubit-cavity interactions have a high ability to generating quantum correlations. The amount of the generated quantum correlation can be improved and accelerated significantly with the increase of the time-dependent qubit location parameter. The increase of the Stark shift ratio and the difference between the mean-number photons of the two cavity fields leads to a clear deterioration of the generated quantum correlation.
\end{abstract}

Keywords: Bell function; skew information quantity; Stark shift; two atoms

\section{INTRODUCTION}

The generation of quantum information resources (as: coherence, entanglement and other types of quantum correlation) due to hybrid nonlinear interactions between two-level atomic systems (qubits) are highly substantial for quantum technologies [1]. Quantum entanglement (QE) is the first type of the quantum correlations between different constituents of multipartite quantum system [2]. In quantum information, $\mathrm{QE}$ is an important physical resource and have various potential applications is several quantum approaches as computing [2], communications [3] and key distribution [4]. Recently, It is emphasized that all quantum correlated states need not be entangled states $[5,6]$. Therefore, there are some quantum information protocols can be implemented with separable mixed states [7]. Consequently, some other quantifiers were introduced to detect QCs beyond entanglement as: measurement-induced disturbance [8], quantum discord [9] and geometrical measures which based on $p$-norm [10] and skew information quantity [11]. QCs can be applied in various branches of quantum engineering, quantum cryptography and quantum information $[2,12,13]$. This work aims at investigating the quantum correlation of uncertainty-induced non-locality (UIN) [14] beyond concurrence entanglement and Bell nonlocality. UIN is primarily defined by the skew information quantity. The UIN has been introduced as another nonclassical quantifier [15-17] and it considered as a revised version of measurement-induced non-locality with contractively.

Research on the quantum information resources present in hybrid nonlinear systems allows the adding of a new physical parameters which contribute to the generation of the quantum correlations beyond entanglement, which are very useful for quantum information [18-21]. The hybrid nonlinear systems can be realized based on the superconducting circuits, ion traps and quantum dots [22]. The hybrid nonlinear qubit-cavity interactions resulted by considering the mode structure of the cavity field (which is leads to atomic motion and Stark shift terms) $[23,24]$ are very important to performance and efficiency in quantum information [24]. In practical experiences, the two-level atomic systems cannot always be regarded to be static during their interaction with the cavity fields. Therefore, the time-dependent two-level atomic location are logically considered. The effect of the time-dependent two-level atomic location has been realized through an atom interacting with a cavity [25]. It is found that The time-dependent qubit-cavity interactions can be enhanced the nonclassicality of the system and the dynamics of entanglement [26-28].

Another important and widely used concept is the entanglement pair coherent state (PCS), which is very useful for primary tasks in quantum information theory [29-31], such as teleportation, Bell nonlocality, and non-classical features of sub-Poissonian distribution and squeezing $[32,33]$. PCSs can be realized by different nonlinear processes in a two-photon medium [32].

The manuscript is organized as follows: The model of the hybrid nonlinear system and its description are presented in the second section. In third and fourth sections, the definition and the dynamics of the atomic non-locality quantifiers (the Bell function, uncertaintyinduced non-locality and concurrence) are illustrated. Finally, in fifth section, the our results are concluded.

* Corresponding author email: eiedkhalil@yahoo.com 


\section{THE CONSIDERED MODEL}

The considered model a hybrid nonlinear system describes a nondegenerate parametric amplifier cavity containing two effective moving atoms (each atom represents qubit with two upper and lower states) with Stark shift interactions [34]. The qubit-field Hamiltonian can be written as

$$
\begin{aligned}
\hat{H}= & \sum_{k=1,2} \omega \hat{N}_{k}+\sum_{k=A, B}\left[\lambda\left(\hat{\Lambda}(t) \hat{\sigma}_{+}^{k}+\hat{\Lambda}^{\dagger}(t) \hat{\sigma}_{-}^{k}\right)\right. \\
& \left.+\frac{1}{2} \omega \hat{\sigma}_{z}^{k}+\left(S_{1} \hat{N}_{1} \hat{\sigma}_{+}^{k} \hat{\sigma}_{-}^{k}+S_{2} \hat{N}_{2} \hat{\sigma}_{-}^{k} \hat{\sigma}_{+}^{k}\right)\right]
\end{aligned}
$$

$\hat{\Lambda}(t)=f(t) \hat{\psi}_{1} \hat{\psi}_{2}$ with the two-field operators $\hat{\psi}_{i}^{\dagger}$ and $\hat{\psi}_{i}(i=1,2)$ and the two-qubit location function described by $f(t)=\cos l t$, where $l$ represents the number of half wavelengths of the cavity field. $\hat{N}_{k}=\hat{\psi}_{k}^{\dagger} \hat{\psi}_{k}$ represent the mean-number photons of the two cavity fields. $\hat{\sigma}_{ \pm}^{k}$ and $\hat{\sigma}_{z}^{(k)}$ represent two-qubit Pauli operators, $\omega$ is the frequency of the qubit-field resonance case. $\lambda$ represents the qubit-field interaction coupling. The Stark shift intensity $S_{i}$ represent the shifting of the energy levels of atoms due to the applied cavity fields [35, 36].

To find a particular solution for the schrodinger equation that describes the dynamics of the atom-cavity state based on Eq.1, we assume that the initial qubit-field state as,

$$
|\psi(0)\rangle=\sum_{n=0}^{\infty} \chi_{n}\left|n+\Delta_{n}, n\right\rangle \otimes\left|1_{A}, 1_{B}\right\rangle,
$$

where $\left|1_{A}, 1_{B}\right\rangle$ denotes to the upper two-qubit states. The two-mode fields are initially in the pair coherent state [32] with the distribution function,

$$
P_{n}=\frac{\alpha^{n}}{N \sqrt{n !\left(n+\Delta_{n}\right) !}}, N^{2}=\sum_{n=0}^{\infty} \frac{|\alpha|^{2 n}}{n !\left(n+\Delta_{n}\right) !},
$$

where $\alpha$ is the eigenvalue of the operator $\hat{\psi}_{1} \hat{\psi}_{2}$, and $|\alpha|^{2}$ represents the initial coherent intensity of the two-mode cavity fields. $\Delta_{n}$ is the difference between the meannumber photons of the two cavity fields.

In the space of the atom-field system, where $\left\{\left|\varpi_{1}\right\rangle=\right.$ $\left|e_{A}, e_{B}\right\rangle \otimes\left|n+\Delta_{n}, n\right\rangle,\left|\varpi_{2}\right\rangle=\left|e_{A}, g_{B}\right\rangle \otimes \mid n+\Delta_{n}+1, n+$ $1\rangle,\left|\varpi_{3}\right\rangle=\left|g_{A}, e_{B}\right\rangle \otimes\left|n+\Delta_{n}+1, n+1\right\rangle,\left|\varpi_{4}\right\rangle=\left|g_{A}, g_{B}\right\rangle \otimes$ $|n+q+2, n+2\rangle\}$, the general solution for the qubit-field state $|\psi(t)\rangle$ takes the form

$$
|\psi(t)\rangle=\sum_{n=0}^{\infty} x_{n}\left|\varpi_{1}\right\rangle+y_{n}\left|\varpi_{2}\right\rangle+z_{n}\left|\varpi_{3}\right\rangle+w_{n}\left|\varpi_{4}\right\rangle .
$$

By using the schrodinger equation, the dynamics of the atom-cavity state are obtained by the following differential equations,

$$
\frac{d}{d t}\left(\begin{array}{c}
x_{n} \\
y_{n} \\
z_{n} \\
w_{n}
\end{array}\right)=\left[\begin{array}{cccc}
0 & \mu_{n} & \mu_{n} & 0 \\
\mu_{n} & \beta_{n} & 0 & \eta_{n} \\
\mu_{n} & 0 & \beta_{n}^{*} & \eta_{n} \\
0 & \eta_{n} & \eta_{n} & 0
\end{array}\right]\left(\begin{array}{c}
x_{n} \\
y_{n} \\
z_{n} \\
w_{n}
\end{array}\right)
$$

where

$$
\begin{aligned}
\mu_{n} & =-i \lambda A(t) \sqrt{\left(n+\Delta_{n}+1\right)(n+1)}, \\
\eta_{n} & =-i \lambda A(t) \sqrt{\left(n+\Delta_{n}+2\right)(n+2)}, \\
\beta_{n} & =-i \lambda A(t)\left[\left(2 n+2 \Delta_{n}+3\right) S-\frac{1}{2 S}(2 n-1)\right],
\end{aligned}
$$

where $S=\sqrt{S_{1} / S_{2}}$ and $A(t)=\frac{1}{l} \sin l t$.

The differential equations of Eq.3 will be calculated numerically. The numerical two-qubit reduced density matrix of the final time-dependent state $|\psi(t)\rangle$ are used to analyze the two-qubit correlations based, which is given by

$$
\hat{\rho}^{A B}(t)=\text { Trace }_{\text {fields }}\{|\psi(t)\rangle\langle\psi(t)|\} .
$$

In the following, the two-qubit correlations will be investigated via the atomic concurrence entanglement, atomic uncertainty-induced non-locality and atomic Bell function non-locality.

\section{TWO-QUBIT CORRELATION QUANTIFIERS}

\section{- Two-qubit concurrence:}

The generated two-qubit entanglement due to the time-dependent qubit-cavity interactions can be investigated by concurrence [38]. The concurrence is one of the most common measure of the entanglement between two particles $A$ and $B$ with the density matrix $\hat{\rho}^{A B}(t)$. The concurrence is defined as

$$
C(t)=\max \left\{0, \sqrt{\mu_{1}}-\sqrt{\mu_{2}}-\sqrt{\mu_{3}}-\sqrt{\mu_{4}}\right\},
$$

where $\mu_{1}>\mu_{2}>\mu_{3}>\mu_{4}$ are the eigenvalues of the matrix: $R=\hat{\rho}^{A B}\left(\sigma_{y} \otimes \sigma_{y}\right) \hat{\rho}^{* A B}\left(\sigma_{y} \otimes \sigma_{y}\right)$.

\section{- Uncertainty-induced non-locality :}

For a bipartite quantum state $\rho^{A B}$ and a local observable $K$, the quantity of the skew information is given by

$$
I\left(\rho^{A B}, K\right)=-\frac{1}{2} \operatorname{Tr}\left[\sqrt{\rho^{A B}}, K\right]^{2},
$$

By using this quantity of the skew information, the uncertainty-induced non-locality (UIN) was introduced as another correlation quantifier to investigate the generated two-qubit correlation. For the two-qubit reduced density matrix $\hat{\rho}^{A B}(t)$, the UIN is given by [14]

$$
U\left(\rho^{A B}(t)\right)=\max _{K} I\left(\rho^{A B}(t), K\right),
$$

that means that, $U\left(\rho^{A B}(t)\right)$ quantifier represents the maximal skew information between the local commuting observable $K$ and the two-qubit 
reduced density matrix. Based on the maximum eigenvalue $\lambda_{\min }$ of the symmetry $3 \times 3$-matrix $W_{A B}=\left[w_{i j}\right]$,

$w_{i j}=\operatorname{Tr}\left\{\sqrt{\rho^{A B}(t)}\left(\sigma_{i} \otimes I\right) \sqrt{\rho^{A B}(t)}\left(\sigma_{j} \otimes I\right)\right\}$,

the explicit form of UIN is given by[14]:

$$
U(t)= \begin{cases}1-\lambda_{\min }\left(W_{A B}\right), & \overrightarrow{\mathbf{r}}=0 \\ 1-\frac{1}{|\overrightarrow{\mathbf{r}}|^{2}} \overrightarrow{\mathbf{r}} W_{A B} \overrightarrow{\mathbf{r}}^{T}, & \overrightarrow{\mathbf{r}} \neq 0\end{cases}
$$

where $\overrightarrow{\mathbf{r}}$ is the Bloch vector and where $\sigma_{i}, i=1,2,3$ are the Pauli matrices.

- Two-qubit Bell non-locality :

Here, we use the maximal values of the two-qubit Bell function $B_{\max }(t)$ as an indicator of two-qubit non-locality $[39,40]$. This two-qubit non-locality satisfies: $B_{\max }(t)>2$, i.e., the Bell-Chsh inequality is violated. The analytical expression of the twoqubit Bell function $B_{\max }(t)$ is given by

$$
B_{\max }(t)=2 \sqrt{\xi_{1}+\xi_{2}}
$$

where $\xi_{i}(i=1,2)$ are the two largest eigenvalues of the matrix $T^{\dagger} T, T=\left[t_{i j}\right]$ is the correlation matrix [40], its elements are defined by $t_{i j}=$ $\operatorname{tr}\left\{\rho_{A B}(t) \sigma_{i}^{(1)} \sigma_{j}^{(2)}\right\}, i, j=1,2,3$. In our investigation, the Bell non-locality is quantified by,

$$
B(t)=B_{\max }(t)-1,
$$

The moving atomic state has Bell non-locality when this function satisfies $B(t)>1$.

\section{ATOMIC NON-LOCALITY DYNAMICS}

We study the effect of time dependence and Stark shift on the measures $C(t), B(t)$ and $U(t)$. Fig.1, displays the generation of the atomic moving non-locality via the Bell function, uncertainty-induced non-locality and concurrence in the presence of the nonlinearity of the Stark shift interaction with different atomic locations. First, we exclude the dependence on time $(l=0)$. For small effective of the Stark shift $S=1$, the quantities $C(t), B(t)$ and $U(t)$ oscillate with the same behavior. The maximum and the minimum of values of entanglement that predicted by both quantifiers are displayed as the same time. The three measures fluctuate chaotically between the maximum and minimum values. The phenomena of sudden death and sudden birth are realized almost every $\frac{\pi}{2}$, as seen in Fig.1a. The random oscillations become regular after adding the time dependence $(l=1)$. Therefore, the entanglement between the two qubits improves
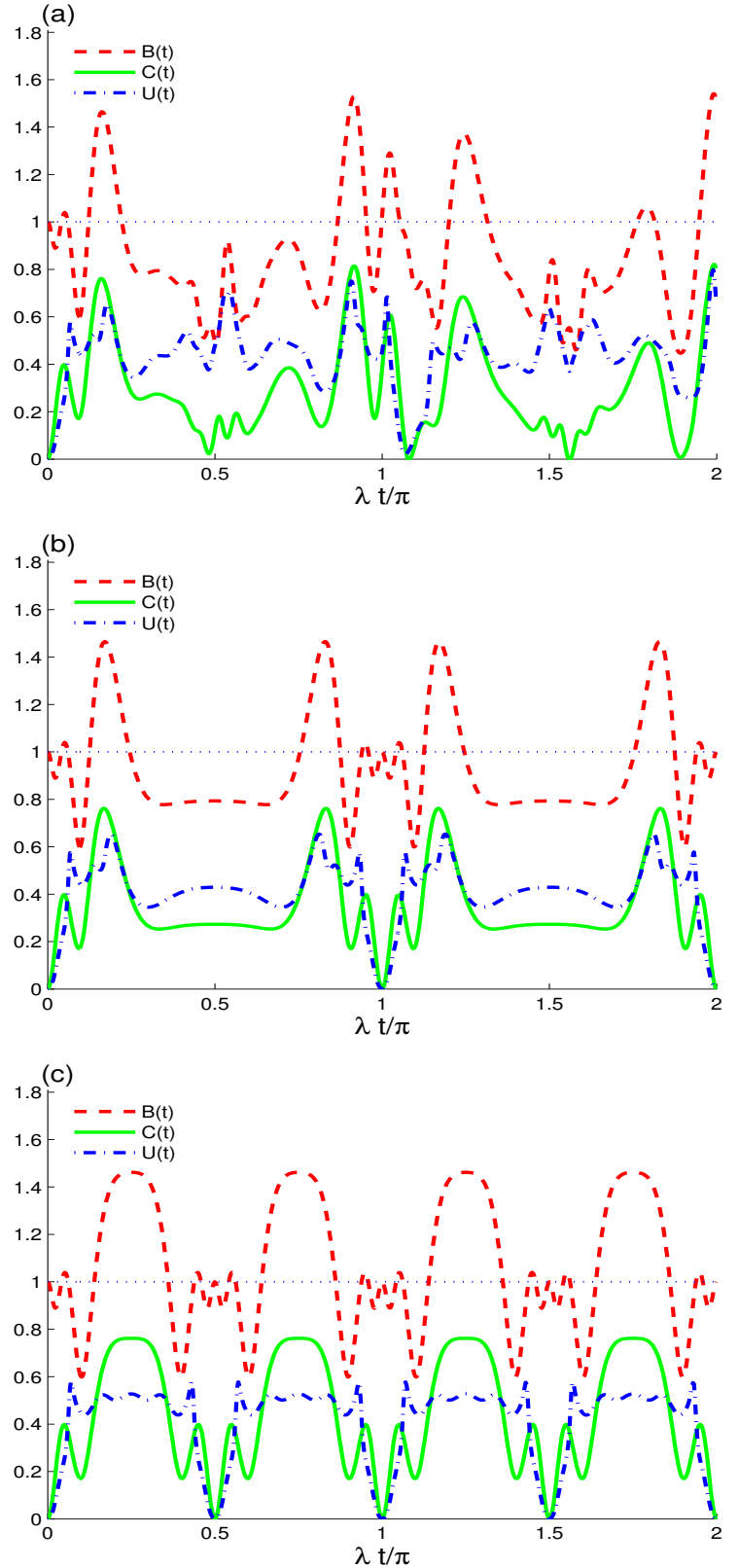

FIG. 1. Dynamics of the non-locality functions for the initial coherent cavity intensity $|\alpha|^{2}=9$ when the mean-number photons of the two cavity fields are equaled $\Delta_{n}=0$ and equal Stark shifts for atomic energy levels $S=1$ with different atomic locations: $l=0$ in (a), $l=1$ in (b), and $l=2$ in (c).

frequently and regularly. The quantities $C(t), B(t)$ and $U(t)$ start from pure state, then partial entanglement is generated until the functions $C(t), B(t)$ and $U(t)$ reach their maximum values. The phenomena of sudden death and sudden birth are realized every $\pi$.

From Fig.1b and c, we can find that the generated quantum correlations between the two qubits improves with increasing $l$ (the time dependence parameter). The intensity of the oscillations increases, so the phenomena 

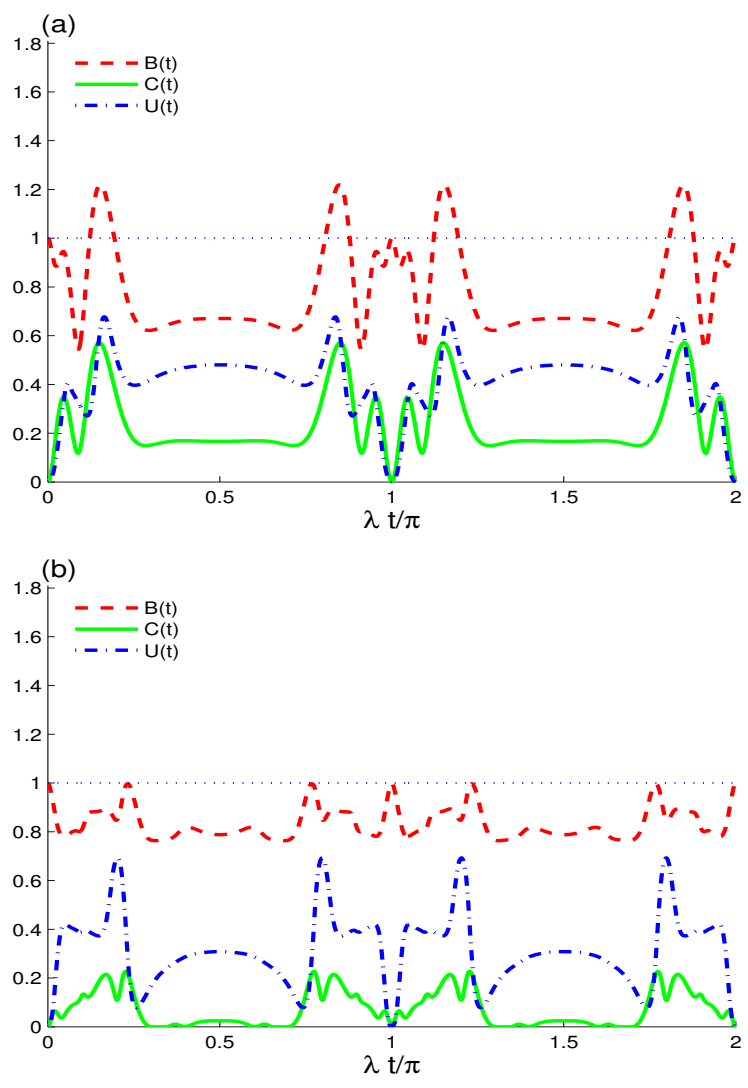

FIG. 2. Temporal evolution of the same non-locality functions considered in Fig.1a, but when the Stark shifts are different: $S=1.5$ in (a) and $S=0.1$ in (b).

of sudden birth and sudden death are realized every $\frac{\pi}{2}$. Moreover, the entanglement periods between the parts of the quantum system improved with increasing the parameter $l$. In general, the upper bounds of the amount of entanglement that shown by the concurrence are much larger than those quantified by the uncertainty-induced non-locality.

In Fig.2, the effect of the Stark shift ratio $S$ on the dynamics of atomic moving non-locality is shown. Consider $S=1.5$, to activate the Stark shift role. Difference of the Stark shift from one reduces the entanglement between the two qubits. We deduce from the results that both $C(t)$ and $B(t)$ are affected by the Stark shift terms, so the maximum values decreased significantly. While the upper bounds of $U(t)$ is not affected by the presence of Stark shift, as evident in Fig.2a.

From Fig.2b we can find that the $C(t)$ and $B(t)$ measures are strongly influenced by increased Stark shift effect. The entanglement between the two qubits is greatly reduced. While the maximum and minimum values of the measure $U(t)$ are almost unaffected by the presence of the Stark shift as seen in Fig.2b. The relationship 9 indicates that the measure $U(t)$ depends on the minimum values of $\rho^{A B}$, so the Stark shift change is related to the
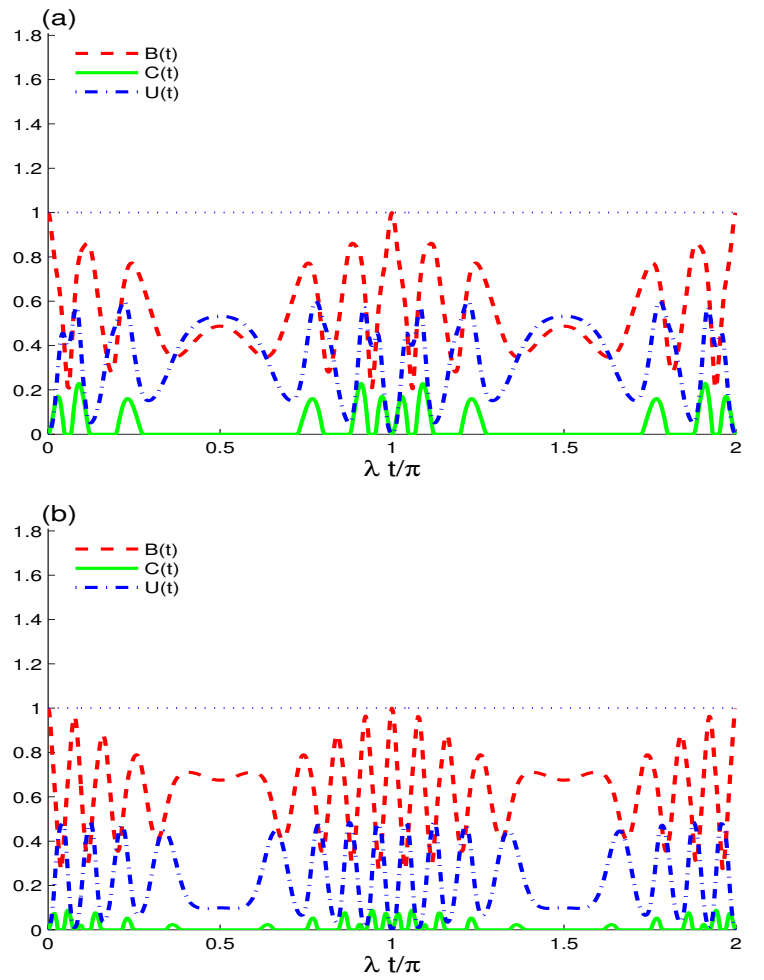

FIG. 3. Time evolution of the same non-locality functions considered in Fig.1a, but for the effect of the difference between the mean photon number of the two cavity fields: $\Delta_{n}=10$ in (a) and $\Delta_{n}=20$ in (b).

maximum values of $\rho^{A B}$. Therefore, the effect of Stark shift on measure $U(t)$ is not demonstrated.

Figs. 3 show the effect of the difference $\Delta_{n}$ between the mean photon number of the two cavity fields on the dynamics of atomic moving non-locality. The amount of entanglement decreases significantly after adding the parameter $\Delta_{n}$. The phenomena of sudden death and sudden birth improve greatly. However, the oscillation amplitudes decrease and consequently, the maximum bounds of the entanglement that predicted via both quantifies decreases. The concurrence measure collapses by increasing the parameter $\Delta_{n}$. The measures $U(t)$ and $B(t)$ are small affected by the increase of the parameter $\Delta_{n}$. We can deduce that the increase of the parameter $\Delta_{n}$ resists the generated atomic correlations.

Fig.4 illustrates the effect of the intensity coherent cavity fields of their mean photon numbers, with the large value for the $\alpha$-parameter, on the dynamics of atomic moving non-locality. Increasing the mean photon numbers (initial coherent intensity of the two-mode cavity fields) leads to increase the generated quantum correlation between the two qubits. We note that the amplitudes and stability of the atomic concurrence entanglement, atomic uncertainty-induced non-locality as well as the atomic Bell non-locality can be enhanced with the increase of the initial coherent intensity of the two-mode 

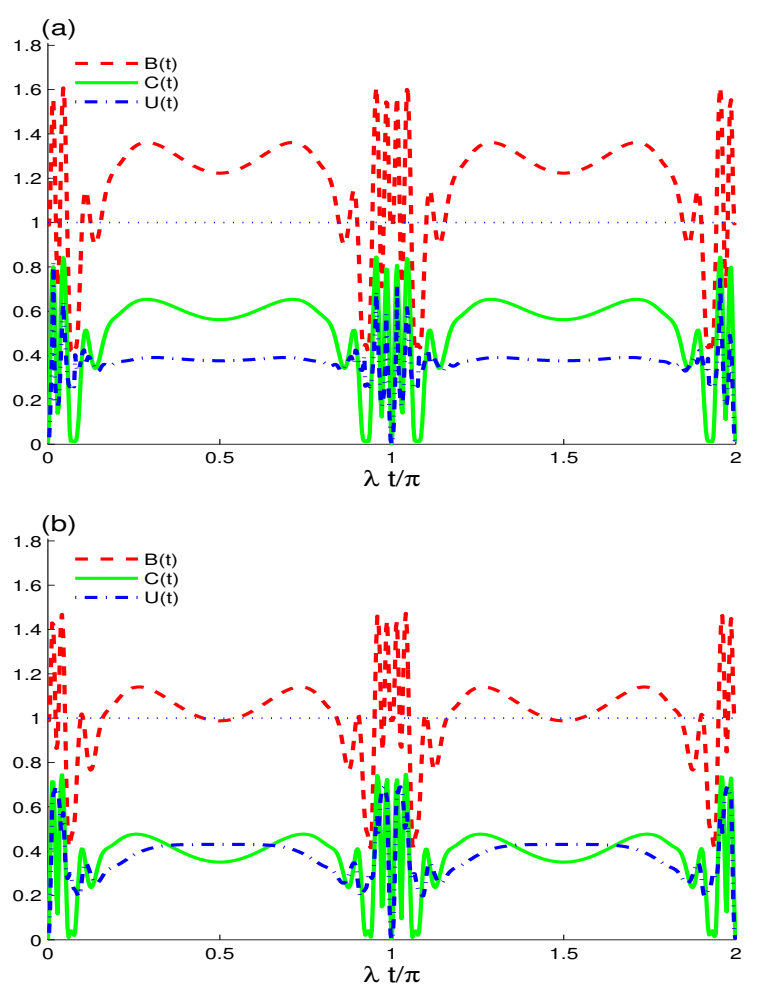

FIG. 4. Time evolution of the same non-locality functions considered in Figs.1b, 2a but with the effect of the mean photon number with $|\alpha|^{2}=225$.

cavity fields.

From Fig.4b, we can find that the quantum correlation measures are affected after considering the Stark shift an increase in the parameter $\alpha$. The Stark shift effect is more pronounced. Whereas, a weak entanglement is generated. The phenomena of sudden death and sudden birth disappear after considering the Stark shift with the exception of some points. Therefore, one can say that the initial coherent intensity of the two-mode cavity fields can be used to a generator factor of the generated quantum correlation between the atomic system.

\section{CONCLUSIONS}

In this paper, we have considered a hybrid nonlinear system describes a nondegenerate parametric amplifier cavity containing two effective moving atoms with Stark shift interactions. The general solution is obtained numerically, to study the effect of the time dependence and the Stark shift parameters on the generated atomic correlations of the Bell function, the uncertainty-induced nonlocality and the concurrence. The results showed that the amount and the stability if the generated atomic correlations have high sensitivity to the parameters of the Stark shift, the qubit-cavity time-dependent coupling, the initial coherent intensity of the two-mode cavity fields. The concurrence entanglement is severely affected when activating the role of Stark shift parameter. The Bellnonlocality, the UIN-correlation, and the entanglement improved significantly with the increase of the qubitcavity time-dependent coupling, which plays an important role in generating quantum correlation between the two systems. Increasing the initial coherent intensity of the two-mode cavity fields generates more entanglement. Whereas, the increase of the parameter of the difference between the mean-number photons of the two cavity fields leads to a clear deterioration of the generated atomic correlations.

\section{REFERENCES}

[1] K. Modi, A. Brodutch, H. Cable, T. Paterek, and V. Vedral, Rev. Mod. Phys. 84, 1655 (2012).

[2] M. A. Nielsen and I. L. Chuang, Quantum Computation and Quantum Information (Cambridge University Press, Cambridge, 2000).

[3] D. Bouwmeester, J. W. Pan, K. Mattle, M. Eibl, H. Weinfurter, A. Zeilinger, Experimental quantum teleportation. Nature 390, 575 (1997)

[4] A. K. Ekert, Quantum cryptography based on Bell's theorem. Phys. Rev. Lett. 67, 661 (1991)

[5] B.P. Lanyon, M. Barbieri, M.P. Almeida, and A. G. White, Phys. Rev. Lett. 101200501 (2008).

[6] A. Datta and G. Vidal, Phys. Rev. A 75042310 (2007).

[7] R. Horodecki, P. Horodecki, M. Horodecki, K. Horodecki, Quantum entanglement. Rev. Mod. Phys. 81, 865 (2009)

[8] S. Luo, Phys. Rev. A 77 (2008) 022301.

[9] H. Ollivier, W. H. Zurek, Phys. Rev. Lett. 88 (2001) 017901.
[10] B. Dakic, V. Vedral, C. Brukner, Phys. Rev. Lett. 105 (2010) 190502.

[11] D. Girolami, T. Tufarelli, G. Adesso, Phys. Rev. Lett. 110, 240402 (2013).

[12] R. Horodecki, P. Horodecki, M. Horodecki, K. Horodecki, Rev. Mod. Phys. 81 (2009) 865; A.-B. A. Mohamed and H. Eleuch, Phys. Scr. 92065101 (2017).

[13] A.-B. A. Mohamed, Eur. Phys. J. D 71261 (2017); A.-B. A. Mohamed,Quantum Inf. Process. 1796 (2018).

[14] S.-X. Wu, J. Zhang, C.-S. Yu, H.-S. Song, Phys. Lett. A 378 (2014) 344.

[15] A.-B. A. Mohamed,and H. Eleuch, J. Opt. Soc. Am. B 35 (2018) 47.

[16] A.-B. A. Mohamed, A. Farouk, M. F. Yassen, and H. Eleuch, Appl. Sci. 10 (2020) 3782.

[17] A.-H Abdel.Aty, H. Kadry, A.-B. A. Mohamed, H. Eleuch, Sci. Rep. 10 (2020) 16640. 
[18] A.-B. A. Mohamed, H. Eleuch, C. H. Raymond Ooi, Sci. Rep.9 19632 (2019).

[19] A.-B. A. Mohamed, H. Eleuch, C. H. Raymond Ooi, Phys. Lett. A 383125905 (2019).

[20] N. Metwally, M. Abdelaty, A.-S.F. Obada, Chaos, Solitons and Fractals 22529 (2004); N. Metwally, M. R. B. Wahiddin and M. Bourennane, Opt. Commun. 257206 (2006)

[21] N. Metwally, M. S. Abdalla, M. Abdel-Aty, Opt. Commun. 2821930 (2009).

[22] H. R. Baghshahia, M. K. Tavassolya, and A. Behjata, Chin. Phys. B 23, 7074203 (2014).

[23] C. V. Sukumar, B. Buck, Phys. Lett. A 83211 (1981).

[24] G.-X. Li, J. S. Peng, Phys. Rev. A 52465 (1995).

[25] G. Rempe, H. Walther, and N. Klein, Phys. Rev. Lett. 58, 353 (1987).

[26] M. Ghorbani, M. J. Faghihi and H. Safari, J Opt. Soc. Am. B 341884 (2017)

[27] D. Xiao-Juan and F. Mao-Fa, Chin. Phys. B 17, 3209 (2008).

[28] A.-S. F. Obada, H.A. Hessian and M. Hashem, Phys. Scr. 81 (2010) 055303.

[29] M. Zukowski, A. Zeilinger, M. A. Horne and A. K. Ekert, Phys. Rev. Lett. 714287 (1993).
[30] C. H. Bennett, D.P. Divincenzo, P.W. Shor, J .A. Smolin, B. M. Terhal and W. K. Wootters, Phys. Rev. Lett., 87 077902 (2001).

[31] L. M. Duan, M. D. Lukin, J. I. Cirac, and P. Zoller, Nature, 414413 (2001).

[32] G. S. Agarwal, A. Biswas, J. Opt. B: Quantum Semiclass. Opt. 7350 (2005).

[33] A.-S.F. Obada, E.M. Khalil, Inter. J. Theor. Phys. 49 (8), 1823 (2010).

[34] A. Salah, A. S. Abdel-Rady, A. N. A. Osman and S. S. Hassan, Sci. Rep. 811633 (2018).

[35] A.-S.F. Obada, M.M.A. Ahmed, E.M. Khalil, S.I. Ali, Opt. Commun. 287215 (2013).

[36] A.-S.F. Obada, E.M. Khalil, M.M.A. Ahmed, J. Mod. Opt. 531149 (206).

[37] N. Metwally, A. Al-Mannai and M. Abdel-Aty, Commun. Theor. Phys. 59 769-772 (2013).

[38] C. H. Bennett, H.J. Bernstein, S. Popescu, B. Schumacher, Phys. Rev. A 53, 2046 (1996); W. K. Wootters, Phys. Rev. Lett. 80 (1998) 2245.

[39] N. Brunner, D. Cavalcanti, S. Pironio, V. Scarani, S. Wehner, Rev. Mod. Phys. 86, 419 (2014)

[40] R. Horodecki, P. Horodecki, and M. Horodecki, Phys. Lett. A 200, (1995) 340. 